PROCEEDINGS OF THE

AMERICAN MATHEMATICAL SOCIETY

Volume 137, Number 10, October 2009, Pages 3343-3353

S 0002-9939(09)09989-4

Article electronically published on May 29, 2009

\title{
SOME ELEMENTARY ESTIMATES FOR THE NAVIER-STOKES SYSTEM
}

\author{
JEAN CORTISSOZ
}

(Communicated by Matthew J. Gursky)

\begin{abstract}
In this paper we study the incompressible Navier-Stokes equations in $\mathbb{T}^{3}=[0,1]^{3}$ with periodic boundary conditions. We show that a weak solution of the Navier-Stokes equations that is small in $L^{\infty}(0, T ; \Phi(2))$ is also smooth. We also give elementary proofs of some classical regularity results for the Navier-Stokes equations involving the Sobolev space $H^{\frac{1}{2}}\left(\mathbb{T}^{3}\right)$.
\end{abstract}

\section{INTRODUCTION}

One of the outstanding problems in mathematics is the existence of global regular solutions to the Navier-Stokes system

$$
\left\{\begin{array}{l}
u_{t}-\Delta u+u \cdot \nabla u+\nabla p=0 \quad \text { in } \quad T^{3} \times(0, \infty) \\
u(x, 0)=\psi, \quad \operatorname{div} u=0
\end{array}\right.
$$

where $T^{3}=[0,1]^{3}$, with periodic boundary conditions.

Several short time existence results and small-initial data-global existence results have been shown for different Banach spaces, and the literature on the subject is extensive.

Given a function $u(x, t) \in L^{2}\left(T^{3}\right)$ we write its Fourier expansion as

$$
\sum_{\mathbf{k}} u_{\mathbf{k}}(t) \exp (2 \pi i\langle x, \mathbf{k}\rangle), \quad \mathbf{k}=\left(k_{1}, k_{2}, k_{3}\right) \in \mathbb{Z}^{3},
$$

and we define the spaces $\Phi(\alpha) \subset \mathcal{P}^{\prime}$ ( $\mathcal{P}^{\prime}$ is the dual of the set of $C^{\infty}$ periodic functions on $T^{3}$ ) as follows:

$$
\Phi(\alpha)=\left\{f:\left|f_{\mathbf{k}}\right| \leq \frac{c}{|\mathbf{k}|^{\alpha}}, \mathbf{k} \neq 0, \quad f_{0}=0\right\}
$$

endowed with the norm

$$
\|f\|_{\alpha}=\sup _{\mathbf{k} \in \mathbb{Z}^{3} \backslash\{0\}}|\mathbf{k}|^{\alpha}\left|f_{\mathbf{k}}\right|,
$$

which makes them Banach spaces. Notice that if $\alpha>\frac{3}{2}, \Phi(\alpha) \subset L^{2}\left(T^{3}\right)$. These spaces are considered in [1, where the following theorem with $\alpha=2+\epsilon, \epsilon>0$, is proved.

Received by the editors October 14, 2008.

2000 Mathematics Subject Classification. Primary 35Q30.

Key words and phrases. Navier-Stokes equations, regularity.

(C)2009 American Mathematical Society Reverts to public domain 28 years from publication 
Theorem 1. Let $0<3 \epsilon<1$ and $\|\psi\|_{\alpha} \leq \delta$ where $\psi=\frac{c_{0}(\mathbf{k})}{|\mathbf{k}|^{\alpha}}$ is the initial condition and $\delta=\delta(\alpha)$ is sufficiently small. Then equation (1) has a global solution $v(t, \mathbf{k})=$ $\frac{c(t, \mathbf{k})}{|\mathbf{k}|^{\alpha}}$ such that $c(t, \mathbf{k})$ is a continuous mapping of $[0, \infty)$ to $L^{\infty}\left(\mathbb{Z}^{3} \backslash\{0\}\right)$.

The result with $\alpha=2$ is announced in [5]. In this note we prove some related results, where the treat for the reader is the elementarity of the proofs which are in the spirit of the ideas presented in [8] (in this note we use nothing beyond the Cauchy-Schwarz inequality).

Namely, we show the following results.

Theorem 2. There exists an $\epsilon>0$ such that if a Leray-Hopf solution $u(x, t)$ of the Navier-Stokes system satisfies

$$
\sup _{|\mathbf{k}| \geq K}|\mathbf{k}|^{2}\left|u_{\mathbf{k}}(t)\right|<\epsilon \quad \text { on } \quad(0, T)
$$

for some $K>0$ independent of time, then $u$ is smooth for every $0<t<T$.

Theorem 3. There exists an $\epsilon>0$ such that if the initial condition $\psi$ satisfies

$$
\|\psi\|_{2}<\epsilon
$$

then any Leray-Hopf solution of (11) with initial condition $\psi$ satisfies

$$
\|u(t)\|_{2}<\epsilon \text { if } t>0 .
$$

Recall that a Leray-Hopf solution of (11) with initial data $\psi \in L^{2}\left(T^{3}\right)$ is a function $u:[0, T) \longrightarrow L^{2}\left(T^{3}\right)$ which is weakly continuous, belongs to the class

$$
L^{\infty}\left(0, T ; L^{2}\left(T^{3}\right)\right) \cap L^{2}\left(0, T ; H^{1}\left(T^{3}\right)\right),
$$

satisfies

$$
\begin{array}{r}
\langle u(t), \varphi(t)\rangle+\int_{0}^{t}-\left\langle u(\tau), \frac{\partial}{\partial \tau} \varphi(\tau)\right\rangle+\langle\nabla u(\tau), \nabla \varphi(\tau)\rangle+\langle u \cdot \nabla u, \varphi\rangle d \tau \\
=\langle\psi, \varphi(0)\rangle
\end{array}
$$

for all $\varphi \in C^{\infty}\left(T^{3} \times[0, T)\right)$ with $\operatorname{div} \varphi=0$, and for which the energy inequality

$$
\|u(t)\|_{L^{2}\left(T^{3}\right)}^{2}+2 \int_{0}^{t}\|\nabla u(\tau)\|_{L^{2}\left(T^{3}\right)}^{2} d \tau \leq\|\psi\|_{L^{2}\left(T^{3}\right)}^{2}
$$

holds.

For the existence of Leray-Hopf solutions with initial data in $L^{2}\left(T^{3}\right)$ the reader may consult Chapter 3 of $[9]$.

An immediate consequence of the existence of a Leray-Hopf solution for a given initial data $u_{0} \in L^{2}\left(T^{3}\right)$, using Theorems 2 and 3 , is the following result.

Corollary 1. There exists an $\epsilon>0$ such that if the initial condition $\psi$ satisfies

$$
\|\psi\|_{2}<\epsilon
$$

then there is a global regular solution to problem (11).

Also, as an application of the methods we use in this paper, we show the following "classical" regularity result. 
Theorem 4. Let $u(x, t) \in L^{\infty}\left(0, T ; H^{\frac{1}{2}}\left(T^{3}\right)\right)$ be a solution of (1). There exists a $\delta>0$ such that for any $\rho>0$, if $\|u(t)\|_{H^{\frac{1}{2}\left(T^{3}\right)}}<\delta$ for $t \in(0, T)$, then there is a $K=K\left(\|u\|_{L^{\infty}\left(0, T ; H^{\frac{1}{2}}\left(T^{3}\right)\right)}, \rho\right)$ such that if $|\mathbf{k}| \geq K$ and $t>\rho$, then

$$
\left|u_{\mathbf{k}}(t)\right| \leq \frac{\left(2(4+\sqrt{2}) L_{0}\right) /\left(1-2 c \sqrt{L_{0}}\right)}{|\mathbf{k}|^{2}}
$$

where $\sqrt{L_{0}}=\sup _{t \in(0, T)}\|u(t)\|_{H^{\frac{1}{2}\left(T^{3}\right)}}$ and $c$ is a universal constant. Therefore if $L_{0}$ is small enough, $u$ is regular on $(0, T)$.

As a consequence of the proof of Theorem 4 one can show that a solution to the Navier-Stokes system that belongs to the space $C\left((0, T), H^{\frac{1}{2}}\left(T^{3}\right)\right)$ is regular (the same result, but with $L^{3}\left(T^{3}\right)$ instead of $H^{\frac{1}{2}}\left(T^{3}\right)$ was proved by Giga in [4] and by von Wahl in [10]), and also that small discontinuities in the $H^{\frac{1}{2}}\left(T^{3}\right)$ norm are allowed (see [6]); we indicate how this can be done at the end of Section 4 . We must point out that the results of this paper can be generalized to arbitrary dimensions (of course with the appropiate hypothesis) without too much effort.

1.1. Some remarks and notation. The Navier-Stokes system can be written in the phase space as follows:

$$
u_{\mathbf{k}, t}^{m}=-|\mathbf{k}|^{2} u_{\mathbf{k}}^{m}-i \sum k_{j} u_{\alpha}^{j} u_{\mathbf{k}-\alpha}^{m}+i \sum \frac{k_{m} k_{l} k_{j}}{|\mathbf{k}|^{2}} u_{\alpha}^{l} u_{\mathbf{k}-\alpha}^{j} .
$$

Notice that by the divergence-free property, one also has that the ODE system for the Fourier coefficients of the Navier-Stokes equation can be written as

$$
u_{\mathbf{k}, t}^{m}=-|\mathbf{k}|^{2} u_{\mathbf{k}}^{m}-i \sum \alpha_{j} u_{\alpha}^{m} u_{\mathbf{k}-\alpha}^{j}+i \sum \frac{k_{m} k_{l} \alpha_{j}}{|\mathbf{k}|^{2}} u_{\alpha}^{l} u_{\mathbf{k}-\alpha}^{j} .
$$

From now on, since all that matters is its asymptotic behavior, we will write the sums

$$
\sum k_{j} u_{\alpha}^{j} u_{\mathbf{k}-\alpha}^{m}=\sum k u_{\alpha} u_{\mathbf{k}-\alpha}, \quad \sum \frac{k_{m} k_{l} k_{j}}{|\mathbf{k}|^{2}} u_{\alpha}^{l} u_{\mathbf{k}-\alpha}^{j}=\sum \mathbf{k} u_{\alpha} u_{\mathbf{k}-\alpha}
$$

and

$$
\sum \alpha_{j} u_{\alpha}^{m} u_{\mathbf{k}-\alpha}^{j}=\sum \alpha u_{\alpha} u_{\mathbf{k}-\alpha}, \quad \sum \frac{k_{m} k_{l} \alpha_{j}}{|\mathbf{k}|^{2}} u_{\alpha}^{l} u_{\mathbf{k}-\alpha}^{j}=\sum \alpha u_{\alpha} u_{\mathbf{k}-\alpha} .
$$

If $X$ is a "classical" Banach space (such as the $L^{p}$ 's or $H^{q}$ 's) we denote its norm by $\|\cdot\|_{X}$. It is also important to notice the following: since the solutions to (1) are divergence free, in our estimations, sums of the form $\sum_{\alpha \in \mathcal{Z}} \alpha u_{\alpha} u_{\mathbf{k}-\alpha}$ are equivalent to sums of the form $\sum_{\alpha \in \mathcal{Z}} \mathbf{k} u_{\alpha} u_{\mathbf{k}-\alpha}$.

\section{Proof of Theorem 3}

Following the analysis and arguments in [8], all we must show is that the sum

$$
\sum_{\alpha} \alpha u_{\alpha} u_{\mathbf{k}-\alpha}
$$

is small compared to the term $-|\mathbf{k}|^{2} u_{\mathbf{k}}$ whenever $\left|u_{\mathbf{k}}\right|$ is close to $\frac{\epsilon}{|\mathbf{k}|^{2}}$. Here we work formally, but the arguments can be made rigorous by using Galerkin approximations 
and then taking limits. Now that the reader has been warned, we proceed with our arguments. Let

$$
\sum_{\alpha} \alpha u_{\alpha} u_{\mathbf{k}-\alpha}=I+I I+I I I
$$

where

$$
I=\sum_{|\alpha| \leq 2|\mathbf{k}|,|\mathbf{k}-\alpha| \leq \frac{|\mathbf{k}|}{2}} \alpha u_{\alpha} u_{\mathbf{k}-\alpha}, \quad I I=\sum_{|\alpha| \leq 2|\mathbf{k}|,|\mathbf{k}-\alpha|>\frac{|\mathbf{k}|}{2}} \alpha u_{\alpha} u_{\mathbf{k}-\alpha}
$$

and

$$
I I I=\sum_{|\alpha|>2|\mathbf{k}|} \mathbf{k} u_{\alpha} u_{\mathbf{k}-\alpha}
$$

Under the assumption $\left|u_{\alpha}\right| \leq \frac{\epsilon}{|\alpha|^{2}}$, we can bound each of these terms as follows:

$$
|I| \leq 2|\mathbf{k}| \frac{4 \epsilon}{|\mathbf{k}|^{2}} \sum_{|\mathbf{k}-\alpha| \leq \frac{|\mathbf{k}|}{2}}\left|u_{\mathbf{k}-\alpha}\right| \leq \frac{4 \epsilon}{|\mathbf{k}|} C \epsilon|\mathbf{k}|=4 C \epsilon^{2},
$$

where $C$ is a constant such that

$$
\sum_{1 \leq|\alpha| \leq r} \frac{1}{|\alpha|^{2}} \leq C \int_{1 \leq|\mathbf{x}| \leq r, \mathbf{x} \in \mathbf{R}^{3}} \frac{1}{|\mathbf{x}|^{2}} d \mathbf{x}
$$

In a similar way, for a constant $C$ universally defined, we obtain the estimate

$$
|I I| \leq 4 C \epsilon^{2} \text {. }
$$

Finally, from

$$
|I I I| \leq|\mathbf{k}| \sum_{|\alpha|>2|\mathbf{k}|} \frac{\epsilon^{2}}{|\alpha|^{2}|\mathbf{k}-\alpha|^{2}}
$$

using the elementary estimates

$$
|\alpha| \leq|\mathbf{k}-\alpha|+|\mathbf{k}| \quad \text { and } \quad|\mathbf{k}-\alpha| \geq|\mathbf{k}| \quad(\text { recall that }|\alpha|>2|\mathbf{k}|),
$$

we obtain

$$
|I I I| \leq|\mathbf{k}| \sum_{|\alpha|>2|\mathbf{k}|} \frac{4 \epsilon^{2}}{|\alpha|^{4}} \leq 4 C \epsilon^{2},
$$

where $C>0$ is a constant such that

$$
\sum_{|\alpha| \geq|\mathbf{k}|} \frac{1}{|\alpha|^{4}} \leq C \int_{|\mathbf{x}| \geq|\mathbf{k}|, \mathbf{x} \in \mathbf{R}^{3}} \frac{1}{|\mathbf{x}|^{4}} d \mathbf{x} \leq \frac{c}{|\mathbf{k}|} .
$$

Therefore, for a constant $C>0$ universally defined, the following bound holds:

$$
\left|\sum_{\alpha} \alpha u_{\alpha} u_{\mathbf{k}-\alpha}\right| \leq 12 C \epsilon^{2}
$$

From the last inequality it is clear that by taking $\epsilon>0$ sufficiently small, the result follows. 


\section{Proof of Theorem 2}

Letting $\rho>0$, we will show that under the hypothesis of Theorem 2, the solution is smooth for $t>\rho$. Define $t_{n}=\rho-\frac{\rho}{2^{n}}$. By the given hypothesis, there is a $k_{-1}$ such that

$$
\left|u_{\mathbf{k}}(t)\right| \leq \frac{\epsilon}{|\mathbf{k}|^{2}} \quad \text { if } \quad|\mathbf{k}| \geq k_{-1}
$$

and

$$
\exp \left(-\frac{\rho|\mathbf{k}|^{2}}{2^{n+1}}\right)<\epsilon^{2^{n}} \quad \text { if } \quad|\mathbf{k}| \geq \frac{k_{-1}}{\epsilon^{2^{n}}} .
$$

Notice that, by the energy inequality satisfied by the Leray-Hopf solutions to the Navier-Stokes equations, there exists a constant $D>0$ such that for all wave numbers $\mathbf{k}$ such that $|\mathbf{k}|<k_{-1}$,

$$
\left|u_{\mathbf{k}}(t)\right| \leq \frac{D}{|\mathbf{k}|^{2}} \quad \text { for } \quad t>0
$$

Now, choose $k_{0}$ so that $\frac{k_{-1}}{k_{0}} \cdot D<\epsilon<\frac{1}{2}$. Define $k_{i}=\frac{1}{\epsilon^{2^{i}}} k_{0}$ and a sequence $\mu_{n}$ inductively as follows:

$$
\mu_{0}=1, \mu_{1}=2, \quad \text { and } \quad \mu_{n+1}=2 \mu_{n}-1 \quad \text { if } \quad n>1 .
$$

The sequence thus defined satisfies the following.

Lemma 1. For the sequence $\mu_{n}$ it follows that $\frac{1}{2}<\frac{\mu_{n}}{2^{n}}<1$, for $n \geq 2$.

Proof. This follows easily by induction.

Now we continue with the proof of Theorem 2. Assume that for $t>t_{n}$ the following estimate holds:

$$
\left|u_{\mathbf{k}}(t)\right| \leq \frac{\epsilon^{\mu_{n}}}{|\mathbf{k}|^{2}} \quad \text { if } \quad|\mathbf{k}| \geq k_{n} .
$$

We are going to show that the estimate improves for $|\mathbf{k}| \geq k_{n+1}$ and times $t>t_{n+1}$. Notice that this estimate holds for $n=0$.

In order to proceed, assume $|\mathbf{k}| \geq k_{n+1}$. As before, we must estimate the sum

$$
\sum_{\alpha} \alpha u_{\alpha} u_{\mathbf{k}-\alpha}=I+I I+I I I+I V+V,
$$

where the meaning of $I, I I, I I I, I V$ and $V$ will become clear in what follows. Before we start, we must point out that the constant $C$ that appears in the following estimates is the maximum between the constant $C$ that appears in inequality (2) and the constant $C$ that appears in inequality (3).

Let us then begin by estimating the first term,

$$
\begin{aligned}
I=I_{1} & +I_{2} \\
\left|I_{1}\right| \leq \sum_{0 \leq|\alpha| \leq k_{-1},|\mathbf{k}-\alpha| \geq \frac{|\mathbf{k}|}{2}\left|\alpha u_{\alpha} u_{\mathbf{k}-\alpha}\right|} & \leq k_{-1} \sum_{0 \leq|\alpha| \leq k_{-1},|\mathbf{k}-\alpha| \geq|\mathbf{k}|}\left|u_{\alpha} u_{\mathbf{k}-\alpha}\right| \\
& \leq \frac{4 k_{-1} \epsilon^{\mu n}}{k_{n+1}^{2}} \sum_{|\alpha| \leq k_{-1}}\left|u_{\alpha}\right| \\
& \leq 4 \epsilon^{\mu_{n}} \cdot D \cdot C \cdot\left(\frac{k_{-1}}{k_{n}}\right)^{2}\left(\frac{k_{n}}{k_{n+1}}\right)^{2}
\end{aligned}
$$

and since $k_{0}<k_{n}$, by our choices we obtain

$$
\left|I_{1}\right| \leq 4 \epsilon^{\mu_{n}} \cdot \frac{1}{2} \cdot C \epsilon^{\mu_{n}}=2 C \epsilon^{2 \mu_{n}} .
$$


On the other hand,

$$
\begin{aligned}
\left|I_{2}\right| & \leq \sum_{k_{-1} \leq|\alpha| \leq k_{n},|\mathbf{k}-\alpha| \geq \frac{|\mathbf{k}|}{2}}\left|\alpha u_{\alpha} u_{\mathbf{k}-\alpha}\right| \\
& \leq k_{n} \sum_{k_{-1} \leq|\alpha| \leq k_{n},|\mathbf{k}-\alpha| \geq \frac{|\mathbf{k}|}{2}}\left|u_{\alpha} u_{\mathbf{k}-\alpha}\right| \\
& \leq \frac{4 k_{n} \epsilon_{n}}{k_{n+1}^{2}} \sum_{k_{-1} \leq|\alpha| \leq k_{n}}\left|u_{\alpha}\right| \\
& \leq 4 \epsilon^{\mu_{n}} \frac{k_{n}}{k_{n+1}^{2}} \cdot C \epsilon k_{n} \leq 2 C \epsilon^{2 \mu_{n}} .
\end{aligned}
$$

Here to go from the second to the third line, we have used the fact that

$$
|\mathbf{k}-\alpha| \geq \frac{|\mathbf{k}|}{2}>\frac{k_{n+1}}{2}>k_{n}
$$

Hence,

$$
|I| \leq\left|I_{1}\right|+\left|I_{2}\right| \leq 2 C \epsilon^{2 \mu_{n}}+2 C \epsilon^{2 \mu_{n}}=4 C \epsilon^{2 \mu_{n}} .
$$

The second term can be estimated as follows:

$$
\begin{aligned}
|I I| \leq \sum_{k_{n} \leq|\alpha| \leq 2|\mathbf{k}|,|\mathbf{k}-\alpha| \geq \frac{|\mathbf{k}|}{2}}\left|\alpha u_{\alpha} u_{\mathbf{k}-\alpha}\right| & \leq \frac{8 \epsilon^{\mu_{n}}}{|\mathbf{k}|} \sum_{k_{n} \leq|\alpha| \leq 2|\mathbf{k}|,|\mathbf{k}-\alpha| \geq|\mathbf{k}|}\left|u_{\alpha}\right| \\
& \leq 16 \epsilon^{\mu_{n}} \cdot C \epsilon^{\mu_{n}}=16 C \epsilon^{2 \mu_{n}} .
\end{aligned}
$$

To estimate the third term we proceed as follows:

$$
\begin{aligned}
|I I I| & \leq \sum_{|\alpha| \leq 2|\mathbf{k}|, k_{n} \leq|\mathbf{k}-\alpha| \leq \frac{|\mathbf{k}|}{2}}\left|\alpha u_{\alpha} u_{\mathbf{k}-\alpha}\right| \\
& \leq \frac{4 \epsilon^{\mu n}}{|\mathbf{k}|} \sum_{|\alpha| \leq 2|\mathbf{k}|, k_{n} \leq|\mathbf{k}-\alpha| \leq \frac{\mathbf{k} \mid}{2}}\left|u_{\mathbf{k}-\alpha}\right| \leq 2 C \epsilon^{2 \mu_{n}}
\end{aligned}
$$

To estimate the fourth term we split it as $I V=I V_{1}+I V_{2}$ and proceed:

$$
\begin{aligned}
\left|I V_{1}\right| & =\sum_{|\alpha| \leq 2|\mathbf{k}|,|\mathbf{k}-\alpha| \leq k_{-1}}\left|\alpha u_{\alpha} u_{\mathbf{k}-\alpha}\right| \\
& \leq \sum_{|\alpha| \leq 2|\mathbf{k}|,|\mathbf{k}-\alpha| \leq k_{-1}} \frac{\epsilon^{\mu_{n}}}{|\alpha|}\left|u_{\mathbf{k}-\alpha}\right| \\
& \leq \frac{2 \epsilon^{\mu_{n}}}{k_{n+1}} \cdot D \cdot C \cdot k_{-1} \leq 2 \epsilon^{\mu_{n}} \frac{k_{n}}{k_{n+1}} \cdot D \cdot C \cdot \frac{k_{-1}}{k_{n}} \leq C \epsilon^{\mu_{n}} \epsilon^{2^{n}} \leq C \epsilon^{2 \mu_{n}},
\end{aligned}
$$

where to go from the first to the second line we have used the fact that $|\mathbf{k}-\alpha| \leq k_{-1}$ implies by the triangle inequality that

$$
|\alpha| \geq|\mathbf{k}|-k_{-1} \geq k_{n+1}-k_{-1} \geq \frac{1}{2} k_{n+1}>k_{n} .
$$

We estimate $I V_{2}$ as follows:

$$
\begin{aligned}
\left|I V_{2}\right| & =\sum_{|\alpha| \leq 2|\mathbf{k}|, k_{-1} \leq|\mathbf{k}-\alpha| \leq k_{n}}\left|\alpha u_{\alpha} u_{\mathbf{k}-\alpha}\right| \\
& \leq \sum_{|\alpha| \leq 2|\mathbf{k}|, k_{-1} \leq|\mathbf{k}-\alpha| \leq k_{n}} \frac{\epsilon^{\mu_{n}}}{|\alpha|}\left|u_{\mathbf{k}-\alpha}\right| \\
& \leq \frac{2 \epsilon^{\mu_{n}}}{k_{n+1}} \cdot C \cdot \epsilon k_{n} \leq 2 \epsilon^{\mu_{n}} C \frac{k_{n}}{k_{n+1}} \epsilon \leq 2 C \epsilon^{\mu_{n}} \epsilon^{2^{n}} \leq 2 C \epsilon^{2 \mu_{n}}
\end{aligned}
$$

where again we have used the fact that $|\mathbf{k}-\alpha| \leq k_{n}$ implies $|\alpha|>k_{n+1}-k_{n}>$ $\frac{1}{2} k_{n+1}$. Hence, we obtain the bound

$$
|I V| \leq\left|I V_{1}\right|+\left|I V_{2}\right|<3 C \epsilon^{2 \mu_{n}} .
$$

Finally, the fifth term yields

$$
\begin{aligned}
|V| & \leq \sum_{|\alpha|>2|\mathbf{k}|}\left|\mathbf{k} u_{\alpha} u_{\mathbf{k}-\alpha}\right| \leq|\mathbf{k}| \sum_{|\alpha|>2|\mathbf{k}|} \frac{\epsilon^{\mu_{n}}}{|\alpha|^{2}} \frac{\epsilon^{\mu_{n}}}{|\mathbf{k}-\alpha|^{2}} \\
& \leq \epsilon^{2 \mu_{n}}|\mathbf{k}| \sum_{|\alpha|>2|\mathbf{k}|} \frac{1}{|\alpha|^{4}} \leq \epsilon^{2 \mu_{n}}|\mathbf{k}| \cdot C \cdot \frac{1}{|\mathbf{k}|} \leq 2 C \epsilon^{2 \mu_{n}}
\end{aligned}
$$

Now let $\epsilon>0$ be small enough (say $\epsilon<\frac{1}{54 C}$ ). Then, for $t>t_{n}$, we obtain

$$
\begin{aligned}
\left|\sum_{\alpha} \alpha u_{\alpha} u_{\mathbf{k}-\alpha}\right| & \leq|I|+|I I|+|I I I|+|I V|+|V| \\
& \leq 4 C \epsilon^{2 \mu_{n}}+16 C \epsilon^{2 \mu_{n}}+2 C \epsilon^{2 \mu_{n}}+2 C \epsilon^{2 \mu_{n}}+3 C \epsilon^{2 \mu_{n}} \\
& =27 C \epsilon^{2 \mu_{n}} \leq \frac{1}{2} \epsilon^{2 \mu_{n}-1}=\frac{1}{2} \epsilon^{\mu_{n+1}} \quad \text { if } \quad|\mathbf{k}| \geq k_{n+1} .
\end{aligned}
$$


Integrating the ODE system for the Fourier coefficients it follows that

$$
\begin{gathered}
\left|u_{\mathbf{k}}(t)\right| \leq \frac{\epsilon^{\mu_{n}}}{|\mathbf{k}|^{2}} \exp \left(-|\mathbf{k}|^{2}\left(t-t_{n}\right)\right)+\frac{1}{2} \epsilon^{\mu_{n+1}}\left(1-\exp \left(-|\mathbf{k}|^{2}\left(t-t_{n}\right)\right)\right) \frac{1}{|\mathbf{k}|^{2}} \\
\text { as long as }|\mathbf{k}| \geq k_{n+1} \quad \text { and } t>t_{n} .
\end{gathered}
$$

This shows that, using (4) and Lemma 1)

$$
\left|u_{\mathbf{k}}(t)\right| \leq \frac{1}{2} \epsilon^{\mu_{n}} \epsilon^{2^{n}} \cdot \frac{1}{|\mathbf{k}|^{2}}+\frac{1}{2} \frac{\epsilon^{\mu_{n+1}}}{|\mathbf{k}|^{2}} \leq \frac{\epsilon^{\mu_{n+1}}}{|\mathbf{k}|^{2}}
$$

if $|\mathbf{k}| \geq k_{n+1}$ and $t>t_{n+1}$.

Assume that $t>\rho>\rho-\frac{\rho}{2^{n}}(n \in \mathbb{N})$. Given any $\mathbf{k}$, let $n \in \mathbb{N}$ be large enough so that $k_{n} \leq|\mathbf{k}|<k_{n+1}$. Then, as we just showed, the estimate

$$
\left|u_{\mathbf{k}}(t)\right| \leq \frac{\epsilon^{\mu_{n}}}{|\mathbf{k}|^{2}} \quad \text { holds. }
$$

Since $\mu_{n} \geq \frac{1}{2} 2^{n}$, it follows that $\epsilon^{\mu_{n}} \leq \frac{k_{0}^{\frac{1}{4}}}{|\mathbf{k}|^{\frac{1}{4}}}$ whenever $|\mathbf{k}| \leq k_{n+1}$, and hence that at time $t>\rho$, for wave numbers $\mathbf{k} \in \mathbb{Z}^{3}$ large enough,

$$
\left|u_{\mathbf{k}}(t)\right| \leq \frac{C}{|\mathbf{k}|^{2.25}} \quad \text { holds. }
$$

This shows that $u \in L^{\infty}\left(\rho, T ; H^{\frac{1}{2}+\frac{1}{8}}\left(T^{3}\right)\right)$, and from the work of Leray ([7]), Theorem 2 follows.

\section{Proof of Theorem 4}

We proceed now with the proof of Theorem 4. Our first important observation is the following simple

Lemma 2. Assume $\left.\left.\int|| \nabla\right|^{\frac{1}{2}} u\right|^{2}<M$. Then there is a constant $c \geq 1$ such that

$$
\sum_{0<|\alpha| \leq r}\left|u_{\alpha}\right| \leq c M^{\frac{1}{2}} r
$$

Proof. The result follows from a judicious application of the Cauchy-Schwarz inequality. Indeed,

$$
\sum_{0<|\alpha| \leq r}\left|u_{\alpha}\right| \leq\left(\sum_{0<|\alpha| \leq r}|\alpha| u_{\alpha}^{2}\right)^{\frac{1}{2}}\left(\sum_{0<|\alpha| \leq r} \frac{1}{|\alpha|}\right)^{\frac{1}{2}} \leq c M^{\frac{1}{2}} r
$$

where $c$ is a constant such that

$$
\sum_{0<|\alpha| \leq r} \frac{1}{|\mathbf{k}|} \leq c \int_{1 \leq|\mathbf{x}| \leq r, \mathbf{x} \in \mathbf{R}^{3}} \frac{1}{|\mathbf{x}|} d \mathbf{x}
$$

Notice that $c$ does not depend on $r$.

Lemma 3. Let $u(x, t) \in L^{\infty}\left(0, T ; H^{\frac{1}{2}}\left(T^{3}\right)\right)$ be a solution of (11). There exists $a \delta>0$ such that for all $\rho>0$, if $\|u(t)\|_{H^{\frac{1}{2}\left(T^{3}\right)}}<\delta$ for $t \in(0, T)$, then there is 
$a K_{0}=K_{0}\left(\|u\|_{L^{\infty}\left(0, T ; H^{\frac{1}{2}}\left(T^{3}\right)\right)}, \rho\right)$ and there is an $N=N\left(\|u\|_{L^{\infty}\left(0, T ; H^{\frac{1}{2}}\left(T^{3}\right)\right)}\right)$ such that if $|\mathbf{k}| \geq K_{0} 2^{n}, n \geq N$, then

$$
\left|u_{\mathbf{k}}(t)\right| \leq \frac{\sqrt{2}(4+\sqrt{2}) L_{0} /\left(1-2 c \sqrt{L_{0}}\right)}{|\mathbf{k}|^{2-\gamma_{n}}}, \quad \gamma_{n}=\frac{1}{2^{n}} \quad \text { if } \quad t \geq t_{n},
$$

where $t_{n}=\rho-\frac{\rho}{2^{n}}$ and $\sqrt{L_{0}}=\sup _{t \in(0, T)}\|u(t)\|_{H^{\frac{1}{2}\left(T^{3}\right)}}$ and $c$ is as in Lemma 2 .

Proof. First, given $\rho>0$ choose $K_{0}$ large enough so that for all $n$, and $l>n$,

$$
\exp \left(-\left(K_{0} \cdot 2^{l}\right)^{2} \cdot \frac{\rho}{2^{n}}\right)<\frac{\sqrt{L_{0}}}{\left(K_{0} 2^{l}\right)^{2}} .
$$

Now we proceed to show estimate (5) by induction. We begin by showing the result for $n=1$. Write

$$
\sum_{\alpha} \alpha u_{\alpha} u_{\mathbf{k}-\alpha}=I+I I,
$$

where the meaning of the terms on the right-hand side will become clear in what follows. We begin by estimating $I$ :

$$
\begin{aligned}
|I| & =\left|\sum_{|\alpha|<2|\mathbf{k}|} \alpha u_{\alpha} u_{\mathbf{k}-\alpha}\right| \leq \sum_{|\alpha|<2|\mathbf{k}|}\left|\alpha u_{\alpha} u_{\mathbf{k}-\alpha}\right| \\
& \leq \sqrt{2} \sqrt{|\mathbf{k}|} \sum_{|\alpha|<2|\mathbf{k}|}\left|\sqrt{|\alpha|} u_{\alpha} u_{k-\alpha}\right| \leq \sqrt{2} \sqrt{|\mathbf{k}|}\|u\|_{H^{\frac{1}{2}}}\|u\|_{L^{2}} .
\end{aligned}
$$

We estimate $I I$ as follows:

$$
\begin{aligned}
|I I| & =\left|\sum_{|\alpha| \geq 2|\mathbf{k}|} \alpha u_{\alpha} u_{\mathbf{k}-\alpha}\right| \\
& \leq \sqrt{2} \sum_{|\alpha| \geq 2|\mathbf{k}|} \sqrt{|\alpha|}\left|u_{\alpha}\right| \sqrt{|\mathbf{k}-\alpha|}\left|u_{\mathbf{k}-\alpha}\right| \leq \sqrt{2}\|u\|_{H^{\frac{1}{2}}}^{2} .
\end{aligned}
$$

Recall that $\sqrt{L_{0}}=\sup _{t \in(0, T)}\|u(t)\|_{H^{\frac{1}{2}}\left(T^{3}\right)}$. Then, from the previous estimates, by integrating the ODE for the Fourier coefficients, we obtain the bound

$$
\left|u_{\mathbf{k}}(t)\right| \leq \sqrt{L_{0}} \exp \left(-\frac{\rho|\mathbf{k}|^{2}}{2}\right)+\frac{\sqrt{2} L_{0}}{|\mathbf{k}|^{\frac{3}{2}}}+\frac{\sqrt{2} L_{0}}{|\mathbf{k}|^{2}} \leq \frac{2 L_{0}}{|\mathbf{k}|^{\frac{3}{2}}}
$$

if $|\mathbf{k}| \geq K_{0}$, for $K_{0}$ large enough, as long as $t>\rho-\frac{\rho}{2}=\frac{\rho}{2}$. Here we have used the fact that for a given $w \in H^{\frac{1}{2}}\left(T^{3}\right)$, if $w_{0}=0$, then $\|w\|_{L^{2}\left(T^{3}\right)} \leq\|w\|_{H^{\frac{1}{2}}\left(T^{3}\right)}$.

Now assume that for $t>t_{n}$ the following estimate holds for $|k| \geq K_{0} 2^{n}$ :

$$
\left|u_{\mathbf{k}}(t)\right| \leq \frac{D_{n}}{|\mathbf{k}|^{2-\gamma_{n}}}
$$

We will show that if $t>t_{n+1}, n \geq 1$, then the following estimate holds:

$$
\left|u_{\mathbf{k}}(t)\right| \leq \frac{D_{n+1}}{|k|^{2-\gamma_{n+1}}} \quad \text { if } \quad|\mathbf{k}| \geq K_{0} 2^{n+1},
$$

where (with $c$ as in Lemma 2)

$$
D_{n+1}=2 c \sqrt{L_{0}} D_{n}+(4+\sqrt{2}) L_{0}, \quad D_{0}=2 L_{0} .
$$

In order to do this, again we must estimate

$$
\sum_{\alpha} \alpha u_{\alpha} u_{\mathbf{k}-\alpha}=I+I I+I I I
$$


where

$$
I=\sum_{|\alpha| \leq 2|\mathbf{k}|,|\mathbf{k}-\alpha| \geq \frac{1}{2}|\mathbf{k}|^{1-\gamma_{n}}} \alpha u_{\alpha} u_{\mathbf{k}-\alpha}, \quad I I=\sum_{|\mathbf{k}-\alpha| \leq \frac{1}{2}|\mathbf{k}|^{1-\gamma_{n}}} \alpha u_{\alpha} u_{\mathbf{k}-\alpha}
$$

and

$$
I I I=\sum_{|\alpha| \geq 2|\mathbf{k}|} \alpha u_{\alpha} u_{\mathbf{k}-\alpha} .
$$

From now on, the assumption $|\mathbf{k}|>K_{0} 2^{n+1}$ is in place. Let us estimate $I$. First notice that if $|\alpha| \leq 2|\mathbf{k}|$ and $|\mathbf{k}-\alpha| \geq \frac{1}{2}|\mathbf{k}|^{1-\gamma_{n}}$, then the inequalities

$$
|\mathbf{k}-\alpha| \geq \frac{1}{2}|\mathbf{k}|^{1-\gamma_{n}} \geq \frac{1}{2}\left(\frac{1}{2}\right)^{1-\gamma_{n}}|\alpha|^{1-\gamma_{n}} \geq \frac{1}{4}|\alpha|^{1-\gamma_{n}},
$$

hold, and from this we deduce that

$$
2 \sqrt{|\mathbf{k}-\alpha|} \geq|\alpha|^{\frac{1}{2}-\gamma_{n+1}}
$$

and hence if $|\alpha| \leq 2|\mathbf{k}|$ we get

$$
\sqrt{|\alpha|} \leq 2|\alpha|^{\gamma_{n+1}} \sqrt{|\mathbf{k}-\alpha|} \leq 2 \cdot 2^{\gamma_{n+1}}|\mathbf{k}|^{\gamma_{n+1}} \sqrt{|\mathbf{k}-\alpha|},
$$

so $I$ can be estimated as

$$
|I| \leq 2 \cdot 2^{\gamma_{n+1}}|\mathbf{k}|^{\gamma_{n+1}} \sum \sqrt{|\alpha|}\left|u_{\alpha}\right| \sqrt{|\mathbf{k}-\alpha|}\left|u_{\mathbf{k}-\alpha}\right| \leq 4 L_{0}|\mathbf{k}|^{\gamma_{n+1}},
$$

where the last inequality follows from Cauchy-Schwarz.

Let us estimate $I I$. From the induction hypothesis, deduce that

$$
|I I| \leq \frac{D_{n}}{\left(1-\frac{1}{2}\right)^{1-\gamma_{n}}|\mathbf{k}|^{1-\gamma_{n}}} \sum_{|\mathbf{k}-\alpha| \leq \frac{1}{2}|\mathbf{k}|^{1-\gamma_{n}}}\left|u_{\mathbf{k}-\alpha}\right| \leq \frac{c \frac{1}{2}}{1-\frac{1}{2}} \sqrt{L_{0}} D_{n}=c \sqrt{L_{0}} D_{n},
$$

since $|\mathbf{k}-\alpha| \leq \frac{1}{2}|\mathbf{k}|^{1-\gamma_{n}}$ implies that

$$
|\alpha| \geq\left(1-\frac{1}{2}\right)|\mathbf{k}|>K_{0} 2^{n}
$$

(i.e., the first inequality follows from the induction hypothesis and the assumption $\left.|\mathbf{k}|>K_{0} 2^{n+1}\right)$. The last inequality in the estimation of $I I$ follows from Lemma 2 ,

Finally, we have that

$$
|I I I| \leq \sum_{|\alpha|>2|\mathbf{k}|} \sqrt{|\alpha|} \sqrt{2|\mathbf{k}-\alpha|}\left|u_{\alpha}\right|\left|u_{\mathbf{k}-\alpha}\right| \leq \sqrt{2} L_{0} .
$$

Hence, integrating the ODE system for the Fourier coefficients yields

$$
\begin{aligned}
\left|u_{\mathbf{k}}(t)\right| \leq & \sqrt{L_{0}} \exp \left(-|\mathbf{k}|^{2}\left(t-t_{n}\right)\right) \\
& +\left(\frac{c \sqrt{L_{0} D_{n}}}{|\mathbf{k}|^{2}}+\frac{\sqrt{2} L_{0}}{|\mathbf{k}|^{2}}+\frac{4 L_{0}}{|\mathbf{k}|^{2-\gamma_{n+1}}}\right)\left(1-\exp \left(-|\mathbf{k}|^{2}\left(t-t_{n}\right)\right)\right),
\end{aligned}
$$

for $|\mathbf{k}| \geq K_{0} 2^{n+1} \quad$ and $\quad t>t_{n}$.

Hence, by our choice of $K_{0}$ (by (6) ), we obtain then the following estimate, valid for all $\mathbf{k}$ such that $|\mathbf{k}| \geq K_{0} 2^{n+1}$ and $t>t_{n+1}$ :

$$
\left|u_{\mathbf{k}}(t)\right|<\frac{2 c \sqrt{L_{0}} D_{n}+(4+\sqrt{2}) L_{0}}{|\mathbf{k}|^{2-\gamma_{n+1}}} .
$$


To finally prove estimate (7), all that is left to show is how to bound the sequence $\left(D_{n}\right)_{n=0,1,2, \ldots}$ effectively for large $n$. This can be done as long as $2 c \sqrt{L_{0}}<1$, and it can be seen in this case that

$$
\lim _{n \rightarrow \infty} D_{n}=\frac{(4+\sqrt{2}) L_{0}}{1-2 c \sqrt{L_{0}}} .
$$

This finishes the proof of the Lemma 3 (of course $\delta$ can be chosen as $\frac{1}{2 c}$ ).

Theorem 4 follows immediatly from Lemma 3 . Indeed,

Proof of Theorem 4. Since for $n$ large enough, $K_{0}^{\gamma_{n}} 2^{\frac{n}{2^{n}}} \leq \sqrt{2}$, if $\mathbf{k}$ satisfies that $K_{0} 2^{n} \leq|\mathbf{k}|<K_{0} 2^{n+1}$ and $t>t_{n+1}$, Lemma 3 yields

$$
\left|u_{\mathbf{k}}(t)\right| \leq \frac{|\mathbf{k}|^{\gamma_{n}} \cdot \sqrt{2}(4+\sqrt{2}) L_{0} /\left(1-2 c \sqrt{L_{0}}\right)}{|\mathbf{k}|^{2}} \leq \frac{2(4+\sqrt{2}) L_{0} /\left(1-2 c \sqrt{L_{0}}\right)}{|\mathbf{k}|^{2}} .
$$

Therefore if $t>\rho$, for wave numbers $\mathbf{k}$ large enough, the required estimate holds, and Theorem 4 is proved.

4.1. Corollary from the proof of Theorem 4. Notice that if there is a $k_{0}$ so that for all $t \in\left(t_{0}, t_{1}\right)$ the expression

$$
\sum_{|\alpha| \geq k_{0}}|\alpha|\left|u_{\alpha}\right|^{2}<\delta \quad \text { for } \delta \quad \text { small enough, }
$$

then it follows from the proof of Theorem 4 that for any $\rho>0$ there is a $K_{1}$ which depends on $\rho$ (but not on $\delta>0$ for small $\delta$ ), such that

$$
\sup _{|\mathbf{k}| \geq K_{1}}|\mathbf{k}|^{2}\left|u_{\mathbf{k}}(t)\right| \leq C \delta \quad \text { for } \quad t \in\left(t_{0}+\rho, t_{1}\right)
$$

and the constant $C$ is also independent of $\delta>0$ for $\delta$ small. From the proof of Theorem 2 it then follows that in the case that (8) holds on $\left(t_{0}, t_{1}\right), u$ is a smooth solution of the Navier-Stokes equation on $\left(t_{0}, t_{1}\right)$. On the other hand, (8) holds whenever $u \in C\left(\left(t_{0}, t_{1}\right), H^{\frac{1}{2}}\left(T^{3}\right)\right)$ (this is a classical result due to Giga in [4] and von Wahl in [10]), or if there is a discontinuity in time at $t=t_{2} \in\left(t_{0}, t_{1}\right)$, then

$$
\limsup _{t \rightarrow t_{2}^{-}}\left\|u(t)-u\left(t_{2}\right)\right\|_{H^{\frac{1}{2}\left(T^{3}\right)}} \quad \text { is small enough. }
$$

This last assertion should be compared with the recent results in [3] and the main result in 6 .

\section{REFERENCES}

[1] M.D. Arnold, Ya. G. Sinai, Global Existence and Uniqueness Theorem for 3D-Navier-Stokes System on $\mathbb{T}^{3}$ for small initial conditions in the spaces $\Phi(\alpha)$, Pure and Applied Mathematics Quarterly 4 (2008), No. 1, 71-79. MR2405995 (2009c:35348)

[2] L. Escauriaza, G. Serëgin, V. Šverák, $L_{3, \infty}$-solutions of Navier-Stokes equations and backward uniqueness, Uspekhi Mat. Nauk 58 (2003), No. 2 (350), 3-44; translation in Russian Math. Surveys 58 (2003), No. 2, 211-250. MR.1992563 (2004m:35204)

[3] A. Cheskidov, R. Shvydkoy, On the regularity of weak solutions of the 3D Navier-Stokes equations in $B_{\infty, \infty}^{-1}$, arXiv:0708.3067v2.

[4] Y. Giga, Solutions for semilinear parabolic equations in $L^{p}$ and regularity of weak solutions of the Navier-Stokes system, J. Differential Equations 62 (1986), No. 2, 186-212. MR833416 (87h:35157) 
[5] The result by V. Kaloshin and Yu. Sannikov is mentioned in Weinan E and Ya. G. Sinai, Recent results in mathematical and statistical hydrodynamics. Russ. Math. Surveys 55 (2000), No. 4, 635-666. MR.1786729(2001h:76032)

[6] H. Kozono and H. Sohr, Regularity criterion of weak solutions to the Navier-Stokes equations, Adv. Differential Equations 2 (1997), No. 4, 535-554. MR1441855 (97m:35206)

[7] J. Leray, Sur le mouvement d'un liquide visqueux emplissant l'espace, Acta Math. 63 (1934), 193-248. MR 1555394

[8] J. Mattingly, Ya. G. Sinai, An elementary proof of the existence and uniqueness theorem for the Navier Stokes equations, Commun. Contemp. Math. 1 (1999), No. 4, 497-516. MR1719695 $(2000 \mathrm{j}: 35226)$

[9] R. Temam, Navier-Stokes Equations and Nonlinear Functional Analysis, CBMS-NSF Regional Conference Series in Applied Mathematics, Society for Industrial and Applied Mathematics (SIAM), Philadelphia, PA, 1983. MR764933 (86f:35152)

[10] W. von Wahl, Regularity of weak solutions of the Navier-Stokes equations, Proc. Symp. Pure Appl. Math., 45, Part 2, Amer. Math. Soc., Providence, RI, 1986, 497-503. MR843635 (87g:35193)

Departamento de Matemáticas, Universidad de los Andes, Bogotá DC, Colombia

E-mail address: jcortiss@uniandes.edu.co 